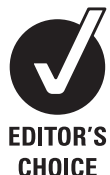

CHOICE

\title{
Role of ambulance response times in the survival of patients with out-of-hospital cardiac arrest
}

\author{
Colin O'Keeffe, ${ }^{1}$ Jon Nicholl, ${ }^{1}$ Janette Turner, ${ }^{1}$ Steve Goodacre ${ }^{2}$
}

${ }^{1}$ Medical Care Research Unit, School of Health and Related Research, University of Sheffield, Sheffield, UK ${ }^{2}$ Emergency Department, Northern General Hospital, Sheffield, UK

\section{Correspondence to} Colin O'Keeffe, Medical Care Research Unit, School of Health and Related Research, University of Sheffield, Regent Court, Regent Street, Sheffield S1 4DA, UK;

c.okeeffe@sheffield.ac.uk

Accepted 14 June 2010 Published Online First 25 August 2010
ABSTRACT

Objectives To evaluate the role of ambulance response times in improving survival for out-of-hospital cardiac arrest (OHCA).

Methods OHCAs were identified by sampling consecutive life-threatening category A emergency ambulance calls on an annual basis for the 5 years 1996/ 7-2000/1 from four ambulance services in England. From these, all calls where an ambulance arrived at the scene and treated or transported a patient were included in the study. These cohorts of patients were followed up to discharge from hospital.

Results Overall, 30 (2.6\%) of the 1161 patients with cardiac arrest survived to hospital discharge. If the patient arrested while the paramedics were on scene, survival to hospital discharge was $14 \%$. The most important predictive factors for survival were response time, initial presenting heart rhythm in ventricular fibrillation and whether the arrest was witnessed. The estimated effect of a 1 min reduction in response time was to improve the odds of survival by $24 \%$ (95\% Cl $4 \%$ to $48 \%)$. The costs of reducing response times across the board by $1 \mathrm{~min}$ at the time of this study were estimated at around $£ 54$ million.

Conclusions The arrival of a crew prior to OHCA means that the chance of surviving the arrest increases sevenfold. Overall it is possible that rapid response to patients in immediate risk of arrest may be at least as beneficial as rapid response to those who have arrested. Concentrating resources on reducing response times across the board to improve survival for those patients already in arrest is unlikely to be a cost-effective option to the UK National Health Service.

\section{INTRODUCTION}

It is well known that survival for patients with out-of-hospital cardiac arrest (OHCA) is dependent on their receiving treatment within a very short time frame. A linear inverse relationship between delay in resuscitation and survival has been documented in a number of studies, and it is generally accepted that people who collapse and are without a pulse for $>12 \mathrm{~min}$ with no intervention are unsalvageable. ${ }^{1}$ Early recognition and access to treatment, early cardiopulmonary resuscitation (CPR) and early defibrillation are all key to survival. Provision of defibrillation by trained paramedics in particular has the potential to improve outcome in sudden cardiac arrest. A Canadian study found a steep decline in survival during the first $5 \mathrm{~min}$ after arrest, with the odds of survival decreasing by $23 \%$ for each additional minute of delay to defibrillation. This suggested that standards for response intervals would produce additional survivors for every minute reduction in response time interval. $^{2}$

Shorter ambulance response times are associated with an increased probability of receiving early defibrillation and subsequent survival, ${ }^{3}$ although this relationship has not always been found. ${ }^{4}$ A number of studies have also reported improvements in survival when the cardiac arrest is witnessed by emergency medical services (EMS) personnel, supporting the concept of rapid EMS response. ${ }^{56}$

Other factors have also been shown to influence survival in OHCA. One study in Sweden identified six factors for increasing the chance of survival (initial rhythm, rapid arrival of the rescue team, place of arrest, witnessed status, bystander CPR and age)..$^{5}$ Perhaps because of differences in the prevalence of these factors, survival rates have been found to differ widely between areas, from $26 \%$ in one US city, ${ }^{7}$ 9\% in Amsterdam, ${ }^{8} 8 \%$ across 10 North American sites, ${ }^{9} 7.8 \%$ in Australia, ${ }^{10} 6.7 \%$ in Italy $^{6}$ and $2 \%$ in Canada ${ }^{11}$ and England. ${ }^{12}$

Evidence showing the importance of early recognition and early access to treatment in survival in OHCA formed the main evidence base for the current ambulance response time standards in England and Wales. ${ }^{13}$ The main performance standard states that $75 \%$ of high priority category A emergency ambulance calls are to be responded to within $8 \mathrm{~min}$. The rationale is that a faster response to life-threatening emergencies, particularly OHCA, could lead to an increase in the number of lives saved.

The purpose of this report is to clarify the relationship between ambulance response times and survival to hospital discharge in OHCA and inform policy for ambulance response times in the UK. It was part of a larger study examining the effect of the introduction of the UK's ambulance service performance standards on outcome in life-threatening category A emergency ambulance calls. ${ }^{14}$

\section{METHODS}

\section{Study design}

The study was part of an evaluation over 5 years into the effect of ambulance response times on patient outcome for a sample of category A life-threatening calls in four ambulance services. The services were included in the study as they had priority dispatch systems in place to categorise calls, were planning to record response times for different categories of calls and represented the range of environment typically encountered in England. Ethical approval for the study was obtained from 24 ethics committees covering the 27 hospitals to which patients could be taken within the geographical boundaries of each of the study ambulance services. 


\section{Case selection}

Consecutive life-threatening calls in which the patient was reported as unconscious or not breathing or with acute chest pain were collected from each ambulance service annually for 5 years, using the emergency medical dispatch codes employed by the four ambulance services to prioritise calls as life-threatening (category A).

\section{Data collection}

Data on response time, defined as the interval from time of the call to time the first ambulance arrived on scene, were collected from routine ambulance service data. All on-scene details recorded in the ambulance patient report form relevant to the OHCAs were collected including observations, treatment, witness and bystander CPR status. If the patient was taken to hospital, the patient's emergency department records and inpatient notes (when admitted) were obtained to determine clinical status and final outcome (whether discharged alive or died).

\section{Identification of cardiac arrests}

In our study we defined possible OHCA as being present if it was detailed on the ambulance patient report form that a patient had no vital signs and CPR was attempted by EMS personnel outside of hospital. Possible cases of OHCA with a non-cardiac diagnosis documented in the emergency department or hospital inpatient notes were excluded from the analysis in accordance with the Utstein style. ${ }^{15}$

\section{Data analysis}

Deaths before arrival at hospital, in the emergency department and in hospital were combined to assess death versus survival to discharge. Survival was examined in relation to whether the arrest was witnessed by EMS crews or other bystanders, the use of bystander CPR and presenting rhythm. For arrests witnessed by non-crew members, these factors were included with ambulance response time in a logistic regression model to estimate the benefit in terms of the reduced odds of deaths of improving response times by $1 \mathrm{~min}$. This estimate of the benefit of reducing response times by 1 min was used in conjunction with published epidemiological, cost and quality of life data in order to estimate the cost to the NHS of reducing ambulance response times by $1 \mathrm{~min}$.

\section{RESULTS}

A total of 1258 patients in whom there was attempted resuscitation were classified by the attending paramedics as having an

Table 1 Clinical diagnosis of out-of-hospital cardiac arrests

\begin{tabular}{lrr}
\hline & N & $\%$ \\
\hline Cardiac disease & & \\
Confirmed myocardial infarction & 46 & 3.7 \\
Other cardiac disease & 28 & 2.2 \\
& & \\
Non-cardiac disease & 7 & 0.5 \\
Injury & 10 & 0.8 \\
Asphyxia & 9 & 0.7 \\
Cerebral vascular accident & 54 & 4.3 \\
Other haemorrhage & & \\
No clinical diagnosis & $1104^{*}$ & 87.8 \\
All & 1258 & 100.0 \\
\hline${ }^{*}$ Of these, 17 had a missing outcome. & &
\end{tabular}

Table 2 Survival by whether the arrest was witnessed by the crew

\begin{tabular}{lcrrr}
\hline & \multicolumn{2}{l}{$\begin{array}{l}\text { Survival to } \\
\text { discharge }\end{array}$} & \multicolumn{1}{c}{ Total } \\
\cline { 2 - 3 } & $\mathbf{n}$ & \% & \multicolumn{1}{c}{ N } \\
\hline Crew witnessed & 8 & 13.8 & 58 \\
Not crew witnessed & 19 & 1.8 & 1054 \\
Not recorded & 3 & 6.1 & 49 \\
Total & 30 & 2.6 & 1161 \\
\hline
\end{tabular}

OHCA. Eighty of these patients had a non-cardiac diagnosis recorded and these patients were excluded from the analysis according to the principles outlined in the Utstein publication (see table 1). ${ }^{15}$ No outcome was found for a further 17 cases, leaving 1161 patients in the main analysis. The median age of the patients was 71 years (IOR 60-79) and just over two-thirds were male (787/1148, 13 not known).

\section{Survival}

Overall, 30 (2.6\%) of the 1161 patients with cardiac arrest survived to hospital discharge.

\section{Crew-witnessed arrests}

Not all of the 1161 patients had an ambulance called because they had arrested. In 58 cases the arrest occurred after the crew arrived at the patient. Eight of these 58 patients survived (13.8\%) compared with just 19 of the 1054 patients (1.8\%) whose arrest was not witnessed by crews (table 2). For a further 49 patients there was no information about whether the arrest was witnessed by the crew.

\section{Rhythms}

An initial rhythm was recorded for 1010 of the 1161 patients, of whom 25 (2.5\%) survived (20 of these were in ventricular fibrillation (VF)). The survival rate for patients whose arrests were witnessed by EMS crews who were found in VF was $25 \%(5 / 20)$ compared with 3.8\% (15/395) for those VF arrests not witnessed by crews (table 3). A total of 170 patients were initially recorded to be in pulseless electrical activity and, in 11 of these cases, the patient was recorded as being in sinus rhythm after receiving $\mathrm{CPR}$, although only 4 survived to hospital discharge.

\section{Bystander intervention}

Of the 1054 patients whose arrests were not witnessed by crews, nearly half (437) were recorded as witnessed by bystanders. Twelve of these patients $(2.8 \%)$ survived compared with only $5 / 559(0.9 \%)$ of those not recorded as witnessed. Overall, 8/240 (3.3\%) of patients survived whose collapse was witnessed and who were given bystander CPR compared with 4/ 190 (2.1\%) who were not given CPR (table 4). For those patients

Table 3 Survival by presenting rhythm

\begin{tabular}{|c|c|c|c|c|c|c|c|c|c|}
\hline \multirow[b]{3}{*}{ Rhythm } & \multicolumn{3}{|c|}{ Crew witnessed } & \multicolumn{3}{|c|}{$\begin{array}{l}\text { Not crew } \\
\text { witnessed }\end{array}$} & \multicolumn{3}{|l|}{ All* } \\
\hline & \multirow[b]{2}{*}{$\mathbf{N}$} & \multicolumn{2}{|c|}{ Survived } & \multirow[b]{2}{*}{$\mathbf{N}$} & \multicolumn{2}{|c|}{ Survived } & \multirow[b]{2}{*}{$\mathbf{N}$} & \multicolumn{2}{|c|}{ Survived } \\
\hline & & $\mathbf{n}$ & $\%$ & & $\mathbf{n}$ & $\%$ & & n & $\%$ \\
\hline VF & 20 & 5 & 25.0 & 395 & 15 & 3.8 & 415 & 20 & 4.8 \\
\hline Asystole & 3 & 0 & 0.0 & 414 & 1 & 0.2 & 417 & 1 & 0.2 \\
\hline PEA & 24 & 2 & 8.3 & 146 & 2 & 1.4 & 170 & 4 & 2.3 \\
\hline Unknown & 11 & 1 & 9.9 & 99 & 1 & 1.0 & 110 & 2 & 1.8 \\
\hline Total & 58 & 8 & 13.8 & 1054 & 19 & 1.8 & 1112 & 27 & 2.4 \\
\hline
\end{tabular}

*For 49 patients there was no information about whether the arrest was witnessed. PEA, pulseless electrical activity; VF, ventricular fibrillation. 
Table 4 Bystander CPR

\begin{tabular}{|c|c|c|c|c|}
\hline \multirow[b]{2}{*}{ Bystander witnessed } & \multirow[b]{2}{*}{ Bystander CPR } & \multicolumn{2}{|c|}{ Survived } & \multirow{2}{*}{$\begin{array}{l}\text { Total } \\
\text { n }\end{array}$} \\
\hline & & n & $\%$ & \\
\hline \multirow[t]{2}{*}{ Yes } & Yes & 8 & 3.3 & 240 \\
\hline & No & 4 & 2.1 & 190 \\
\hline \multirow[t]{2}{*}{ No } & Yes & 2 & 1.1 & 188 \\
\hline & No & 3 & 0.8 & 366 \\
\hline \multirow[t]{2}{*}{ Not known } & Yes & 1 & 8.3 & 12 \\
\hline & No & 0 & 0.0 & 32 \\
\hline
\end{tabular}

CPR, cardiopulmonary resuscitation.

receiving bystander $\mathrm{CPR}$ who were found in VF at the time of EMS contact, $7 / 129$ (5.4\%) survived.

\section{Response times}

In the 1054 patients whose arrests were not witnessed by crews after they had arrived on scene, 19 patients (1.8\%) survived. The survival rates by response time are shown in figure 1 . Of these 19 survivors, $15(79 \%)$ had a response time of $\leq 8 \mathrm{~min}$ and $12(63.2 \%)$ were responded to by a crew within 6 min. However, 4 (21\%) had a recorded response time of $8-12 \mathrm{~min}$. Examining the effect of response time in patients found in VF (figure 2) shows that short response times ( $<6 \mathrm{~min}$ ) can lead to a survival chance of $>5 \%$. Of those patients in VF who were attended within 6 min and who also had bystander CPR, 9/62 (14.5\%) survived (figure 3).

Factors associated with survival in OHCA not witnessed by crew Including all the factors and the patient's age in a multiple logistic regression model, the most important predictive factors for survival were age, response time and whether or not in VF (table 5). The estimated effect of a reduction in response time of 1 min was to improve the odds of survival by $24 \%$ ( $95 \%$ CI $4 \%$ to $48 \%$ ).

\section{Estimate of quality-adjusted life years}

A reduction in response times of $1 \mathrm{~min}$ was estimated to improve the odds of survival by $24 \%$ (in effect increasing survival from our reported rate of $2.6 \%$ to $3.2 \%$ ). The most recent data on the incidence of OHCAs in England and Wales reported that resuscitation was attempted in 24936 cases. ${ }^{16}$ An increased survival rate of $3.2 \%$ would therefore translate into approximately 149 more survivors in OHCA per year. Assuming that the survivors live for an average of 5.61 years after discharge $e^{17}$ with a quality of life utility of $0.72,{ }^{18} 19$ then on average each survivor would achieve 3.72 quality-adjusted life years (after discounting at 3.5\%).

\section{Costs}

Data collected by a typical English ambulance service in 1998 suggested that a reduction in average ambulance response times

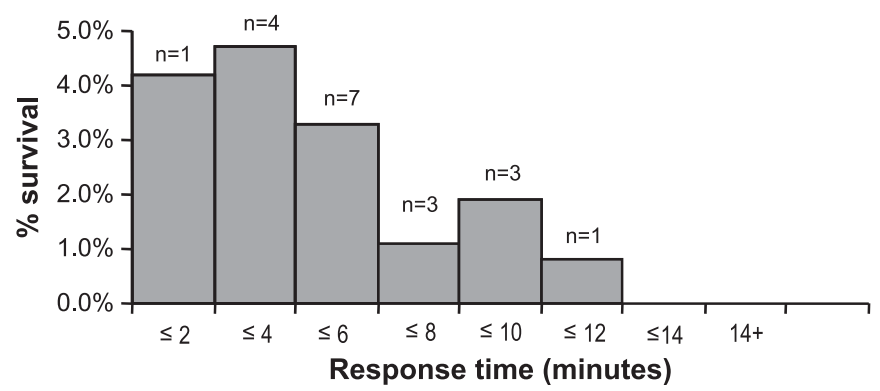

Figure 1 Survival in out-of-hospital cardiac arrest by response time.

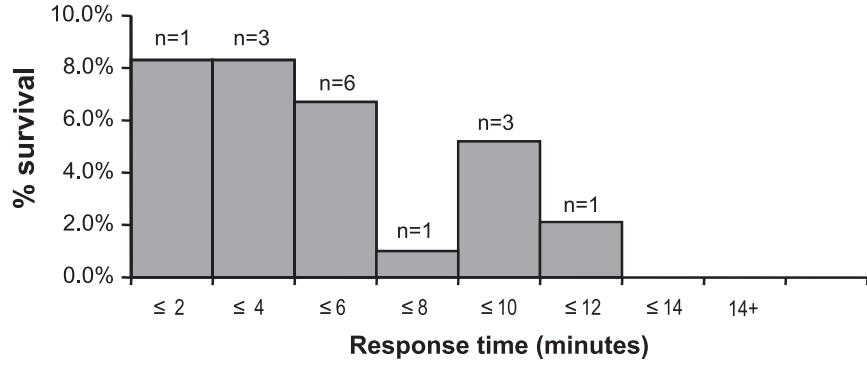

Figure 2 Survival in out-of-hospital cardiac arrest of patients in ventricular fibrillation by response time.

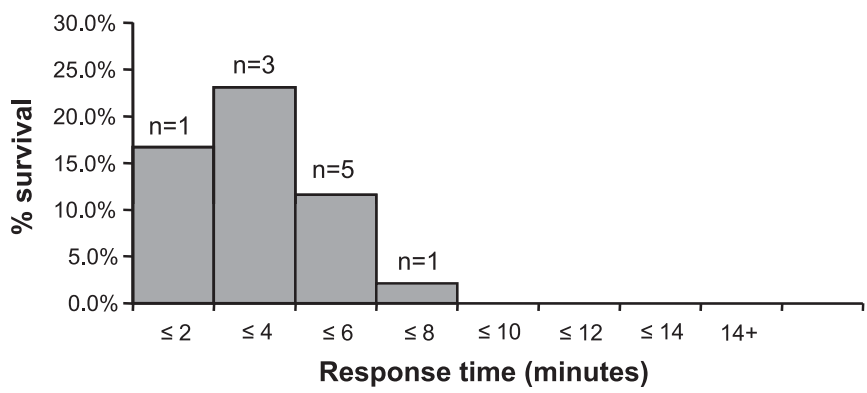

Figure 3 Survival of patients with out-of-hospital cardiac arrest found in ventricular fibrillation who had bystander cardiopulmonary resuscitation by response time.

of $1 \mathrm{~s}$ would cost $£ 28000$ per year. ${ }^{20}$ According to these study figures, a reduction in average response times of 1 min would therefore mean an annual cost of $£ 1.68$ million to that service alone. At the time our study, which was before the merger of ambulance service trusts, this would have represented a cost to the NHS in England and Wales of approximately $£ 54$ million (based on 32 ambulance trusts).

\section{DISCUSSION}

We have examined the influence of possible predictive factors on survival in patients with OHCA in a large unselected cohort of routinely managed patients with cardiac arrest. Overall, 30 $(2.6 \%)$ of the 1161 patients with cardiac arrest survived to hospital discharge. Our figure of $2.6 \%$ is lower than some UK studies $^{21-23}$ but corresponds with another. ${ }^{12}$ We did not exclude patients in our study in whom resuscitation was terminated in the community or who were dead on arrival at hospital, as in some UK studies. ${ }^{21-23}$ Our comprehensive unselected cohort may provide a more accurate estimate of survival in cardiac arrest in routine practice. The estimated effect of a reduction in response time of $1 \mathrm{~min}$ is to improve the odds of survival by $24 \%$ (95\% CI $4 \%$ to $48 \%$ ). This closely replicates an earlier study which found an increase in the odds of survival of $23 \%$ for a 1 min reduction in response time..$^{2}$

Table 5 Odds of survival in out-of-hospital cardiac arrest

\begin{tabular}{llll}
\hline Factor & $\begin{array}{l}\text { Estimated OR } \\
\text { for survival }\end{array}$ & $\mathbf{9 5 \%} \mathbf{C l}$ & p Value \\
\hline Age (per year older) & 0.97 & 0.95 to 1.0 & 0.03 \\
Rhythm (VF vs other) & 5.57 & 1.44 to 21.6 & 0.01 \\
Response time (per min shorter) & 1.24 & 1.04 to 1.48 & 0.02 \\
$\begin{array}{l}\text { Bystander-witnessed arrest } \\
\text { (witnessed vs not witnessed) }\end{array}$ & 1.64 & 0.52 to 5.19 & 0.39 \\
Bystander CPR vs not & 1.32 & 0.46 to 3.80 & 0.60 \\
\hline
\end{tabular}

$\mathrm{CPR}$, cardiopulmonary resuscitation; $\mathrm{VF}$, ventricular fibrillation. 
The association between response time and survival shown in our study was expected, and the value of our analysis lies in quantifying this effect and assessing the implication for ambulance response time policy in the UK. Using our data in conjunction with published data, we have estimated that a 1 min reduction in response times would save approximately 149 lives per year and that each survivor would achieve 3.72 quality-adjusted life years. The UK NHS is currently willing to afford no more than approximately $£ 20000-30000$ per qualityadjusted life year for health care, ${ }^{24}$ which equates to spending approximately $£ 11$ million to $£ 17$ million to reduce response time targets by 1 min across the board based on saving 149 lives per annum. According to our cost analysis, we estimate the cost to the NHS in England and Wales of this 1 min reduction in response times at approximately $£ 54$ million. This suggests that reducing ambulance response times across the board in order to save the lives of people with OHCA may not represent a costeffective policy unless there are substantial additional benefits.

Shorter response times are increasingly important in survival in OHCA if other predictive factors are also present in patients. In patients in VF who have already arrested and whose collapse is witnessed by bystanders, a rapid response within a few minutes results in $5-10 \%$ of patients being resuscitated and surviving to discharge. In addition, if these patients receive CPR, a rapid response may produce results that are better than this. Of course it is not possible to selectively target a quicker response to these particular groups of OHCA patients.

We have found that the arrival of a crew prior to OHCA means that the chance of surviving the arrest increases sevenfold. Paramedics are also able to prevent arrest, for example, by using prehospital thrombolysis. A quick response to patients with severe acute crushing chest pain may enable rapid reperfusion preventing arrest in some patients and resuscitation in $14 \%$ of those who do arrest in the presence of the crew. Overall it is possible that rapid response to patients in immediate risk of arrest may be at least as beneficial as rapid response to those who have arrested. Other approaches to improving survival from cardiac arrest that may be more cost-effective than reducing response times include more emphasis on the quality of care provided by paramedics at the scene, greater public access to defibrillators, public training in basic life support ${ }^{25}$ and secondary prevention in patients with established cardiovascular disease to prevent OHCA.

\section{Limitations}

All data collected in this study including response times were calculated from routine data collected by the four ambulance services included in the study and these data were of variable quality. For the purposes of our study we assumed that the times recorded in the ambulance service data were valid. However, discrepancies in the way UK ambulance services record times have been reported. ${ }^{26}$ It is possible that incorrect recording of times occurred in our data, artificially improving our reported response time results. Estimated costs of reducing ambulance response times by $1 \mathrm{~min}$ in this study are based on data collected before the merger of ambulance services in 2006. Our primary endpoint was survival to hospital discharge and we were therefore unable to assess quality of life in our cohort of survivors, although estimates were made for our cohort using data from previous case reviews.

Funding The study was funded by the Department of Health, and the MCRU is supported by the Department of Health. However, the views expressed are those of the authors alone.

\section{Competing interests None.}

Ethics approval This study was conducted with the approval of the 24 local research ethics committees.

Provenance and peer review Not commissioned; externally peer reviewed.

\section{REFERENCES}

1. Cummins R, Ornato J, Thies W, et al. Improving survival from sudden cardiac arrest: the "chain of survival" concept. A statement for health professionals from the advanced life support committee and the emergency cardiac care committee, American Heart Association. Circulation 1991:83:1832-47.

2. De Maio V, Stiell I, Wells G, et al. Optimal defibrillation response intervals for maximum out of hospital cardiac arrest survival rates. Ann Emerg Med 2003:42:242-50

3. Pell J, Sirel J, Marsden A, et al. Effect of reducing ambulance service response times on deaths from out of hospital cardiac arrest: a cohort study. BMJ 2001;322:1385-8.

4. Finn J, Jacobs I, Holman C, et al. Outcome of out of hospital cardiac arrest patients in Perth, Western Australia, 1996-1999. Resuscitation 2001;51:247-55.

5. Herlitz J, Engdahl J, Svensson L, et al. Factors associated with an increased chance of survival among patients suffering out of hospital cardiac arrest in a national perspective in Sweden. Am Heart J 2005;149:61-6.

6. Kette F, Sbrojavacca R, Rellini G, et al. Epidemiology and survival rate of out of hospital cardiac arrest in North East Italy: the F.A.C.S. study. Fruili Venezia Guilia Cardiac Arrest Co-operative Study. Resuscitation 1998;36:153-9.

7. Eisenberg $\mathbf{M}$, Horwood B, Cummins R, et al. Cardiac arrest and resuscitation: a tale of 29 cities. Ann Emerg Med 1990;19:179-86.

8. Waalewijn R, de Vos R, Koster R. Out of hospital cardiac arrests in Amsterdam and its surrounding areas: results from the Amsterdam resuscitation study (ARREST) in Utstein style. Resuscitation 1998;38:157-67.

9. Nichol G, Thomas E, Callaway C, et al. Regional variation in out-of-hospital cardiac arrest incidence and outcome. JAMA 2008;300:1423-31.

10. Fridman $\mathbf{M}$, Barnes $\mathbf{V}$, Whyman $\mathrm{A}$, et al. A model of survival following pre-hospital cardiac arrest based on the Victoria Ambulance Cardiac Arrest Register. Resuscitation 2007:75:311-22.

11. Brison R, Davidson J, Dreyer J, et al. Cardiac arrest in Ontario: circumstances, community response, role of prehospital defibrillation and predictors of survival. CMAJ 1992;147:1427-8.

12. Wright D, Bannister J, Ryder $\mathrm{M}$, et al. Comparison of two methods of transporting paramedics to cardiac arrests outside hospital. Resuscitation 1992;23:193-7.

13. Chapman R. Review of Ambulance Performance Standards. Final Report of Steering Group. London: NHS Executive, 1996.

14. Turner J, O'Keeffe C, Dixon S, et al. The costs and benefits of changing ambulance service response time performances. A report to the Department of Health. Medical Care Research Unit. Sheffield: University of Sheffield, 2006.

15. Cummins RO, Chamberlain DA, Abramson NS, et al. Recommended guidelines for uniform reporting of data from out of hospital cardiac arrest: the Utstein style. A statement for health professionals from a taskforce of the American Heart Association, the European Resuscitation Council, the Heart and Stroke Foundation of Canada and the Australian Resuscitation Council. Circulation 1991;84:960-75

16. The Ambulance Association and Joint Royal College Ambulance Liaison Committee (JRALC) Clinical Effectiveness Committee. National Out of Hospital Cardiac Arrest Project. August 2005.

17. Eisenberg MS, Hallstrom A, Bergner L. Long term survival after out-of-hospital cardiac arrest. N Engl J Med 1982;306:1340-3

18. Nichol G, Stiell IG, Herbert $P$, et al. What is the quality of life of survivors of cardiac arrest? A prospective study. Acad Emerg Med 1999;6:95-102.

19. Beck JR Jr, Pauker SG, Gottlieb JE, et al. A convenient approximation of life expectancy (the 'DEALE), II: use in medical decision making. Am J Med 1982; 73:889-97

20. Fischer AJ, O'Halloran P, Littlejohns P, et al. Ambulance economics. J Public Health Med 2000:22:413-21.

21. Rainer TH, Gordon MWG, Robertson CE, et al. Evaluation of outcome following cardiac arrest in patients presenting to two Scottish emergency departments. Resuscitation 1995;29:33-9.

22. Hassan TB, Hickey FG, Goodacre S, et al. Prehospital cardiac arrest in Leicestershire: targeting areas for improvement. J Accid Emerg Med 1996;13:251-5.

23. Moore MJ, Glover BM, McGann CJ, et al. Demographic and temporal trends in out of hospital sudden cardiac death in Belfast. Heart 2006;92:311-15.

24. National Institute for Health and Clinical Excellence. Guide to the methods of technology appraisal. London: NICE, 2008.

25. Davies CS, Colquhoun MC, Boyle R, et al. A national programme for on-site defibrillation by lay people in selected high risk areas: initial results. Heart 2005;91:1299-302.

26. The Information Centre. Ambulance services 2005-6. August 2006. 